Technological University Dublin

2011-05-14

\title{
Studies of Shrinkage as a Result of Holographic Recording in Acrylamide Based Photopolymer Film
}

\author{
Mohesh Moothanchery \\ Dublin Insitute of Technology, moheshm@gmail.com \\ Izabela Naydenova \\ Technological University of Dublin, izabela.naydenova@tudublin.ie \\ Vincent Toal \\ Technological University of Dublin, vincent.toal@tudublin.ie
}

Follow this and additional works at: https://arrow.tudublin.ie/cieoart

Part of the Engineering Commons, and the Optics Commons

\begin{abstract}
Recommended Citation
Moothanchery, M., Naydenova, I., Toal, V. (2011) Studies of shrinkage as a result of holographic recording in acrylamide-based photopolymer film, Applied Physics A: Materials Science and Processing. Vol. 104, 3, pp .899-902. doi:10.1007/s00339-011-6435-8
\end{abstract}

This Article is brought to you for free and open access by the Centre for Industrial and Engineering Optics at ARROW@TU Dublin. It has been accepted for inclusion in Articles by an authorized administrator of ARROW@TU Dublin. For more information, please contact arrow.admin@tudublin.ie, aisling.coyne@tudublin.ie, gerard.connolly@tudublin.ie.

Funder: Strand I-Post-Graduate R\&D Skills Programme

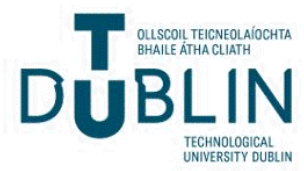




\title{
Studies of shrinkage as a result of holographic recording in acrylamide based photopolymer film
}

\author{
Mohesh Moothanchery, Izabela Naydenova \& Vincent Toal \\ Centre for Industrial \& Engineering Optics \\ Dublin Institute of Technology \\ Dublin 8, Ireland \\ mohesh.moothanchery@student.dit.ie \\ Ph: (+353-1)4024702, Fax: (+353-1)4024988
}

\begin{abstract}
We studied the shrinkage in acrylamide based photopolymer by measuring the Bragg detuning of transmission diffraction gratings recorded at different slant angles and at different intensities. Transmission diffraction gratings of spatial frequency 1000 lines/mm were recorded in an acrylamide based photopolymer film having $60 \pm 5 \mu \mathrm{m}$ thickness. We have obtained the grating thickness and the final slant angles from the Bragg curve and hence calculated the shrinkage caused by holographic recording. The shrinkage of the material was evaluated for three different recording intensities 1,5 and $10 \mathrm{~mW} / \mathrm{cm}^{2}$, while the total exposure energy was kept constant at $80 \mathrm{~mJ} / \mathrm{cm}^{2}$. From the experimental results it can be seen that the shrinkage of the material is higher for recording with lower intensities and the corresponding values are $1.9 \%, 1.3 \%$ and $1 \%$.
\end{abstract}

\section{Introduction}

Photopolymers undergo modulation of refractive index upon exposure to interference patterns and have been widely developed because of their easy processing, high light sensitivity, high refractive index contrast and low cost. This makes photopolymers a good prospect for the manufacture of holographic diffractive elements, as media for holographic data storage and for security holograms, etc $[1,2]$. The material investigated in this study is an acrylamide based photopolymer developed at the Centre for Industrial and Engineering Optics having higher diffraction efficiency and refractive index modulation [3]. The shrinkage was studied by angular selectivity for various slant angles. Only a few attempts have been made to provide clear information of what is going on inside the polymer material $[8,9]$. It is known that the process of monomer diffusion plays an important role in holographic recording [11] and it would be useful to evaluate the relation between Bragg shift occurring due to shrinkage and recording intensities governing the polymerization rate and the amount of the concentration gradient driven diffusing species. One challenge with photopolymers is that the material shrinks during polymerisation leading to Bragg mismatch at read-out [2]. This change is due to a 
change in fringe spacing in the thickness direction, hence out of plane and shrinkage has not been observed in other directions [4]. Such changes can be observed in slanted transmission and reflection gratings [5-6], but in the case of an unslanted grating the fringes are perpendicular to the surface of the material and neither the spacing of the recorded fringes nor the slant angle [7] are affected. We obtained the Bragg curves of the slanted holographic gratings after recording and evaluated the angular shifts of the peaks by curve fitting using Kogelnik's coupled wave theory as explained in [10]. We can write the expression for the fractional change $\Delta d$ in grating thickness $d$ by knowing the initial and final slant angles as explained in [7].

$$
\frac{\Delta d}{d}=\frac{\tan \phi_{1}}{\tan \phi_{0}}-1
$$

where $\phi_{0}$ and $\phi_{1}$ are the initial and final slant angles. Hence the shrinkage can be calculated.

\section{Experimental procedures}

\subsection{Sample preparation}

The photopolymer layer was prepared as previously described [3]. Briefly, 0.6g of acrylamide monomer was added to $17.5 \mathrm{ml}$ stock solution of polyvinyl alcohol $(10 \% \mathrm{wt})$. Then $2 \mathrm{ml}$ of triethanolamine was added followed by $0.2 \mathrm{~g}$ of $N, N_{-}$Methylenebisacrylamide and finally $4 \mathrm{ml}$ of Erythrosin B dye ( $0.11 \mathrm{wt} \%$ stock solution). Of this solution, $0.6 \mathrm{ml}$ was spread on a $25 \mathrm{~mm} \times 75 \mathrm{~mm}$ glass plate. The samples were dried for $24 \mathrm{~h}$. Sample thickness after drying was approximately $60 \pm 5 \mu \mathrm{m}$.

\subsection{Experimental setup}

The basic setup used to record holographic gratings is shown in Fig.1. The 532-nm beam from an $\mathrm{Nd}-\mathrm{YVO}_{4}$ laser was spatially filtered, expanded to $1 \mathrm{~cm}$ diameter and collimated. The beam was then split into two using a beamsplitter. The two beams of the same intensity were made to overlap at the sample of photopolymer at an angle of $30.85^{\circ}$, so the spatial frequency was approximately $1000 \mathrm{lines} / \mathrm{mm}$. The photopolymer layer was mounted on a high-precision computer-controlled rotational stage (Newport M-URM100ACC) so that the normal to the layer bisected the interbeam angle. This ensured that the 
beam ratio was constant across the illuminated area and that the grating was unslanted. The whole holographic setup was placed on a vibration free optical table. Gratings were recorded using intensities of 1,5 and $10 \mathrm{~mW} / \mathrm{cm}^{2}$ and exposure times 80, 16 and $10 \mathrm{~s}$. The diffraction efficiencies of the gratings were monitored using a He-Ne laser which was diffracted by the recorded grating but not absorbed by the photopolymer.

The intensity of the He-Ne laser incident on the grating and the diffracted intensity were both measured using a power meter (Newport Optical Power Meter .Model 1830-C). It was necessary to ensure that the He-Ne laser was incident at the Bragg angle for 633-nm light even though there will be a small change in the position of maximum diffraction intensity for the unslanted gratings due to imperfections.

After recording the unslanted gratings and obtaining the angular selectivity profiles we also measured the profiles of gratings recorded with slant angles of $-10^{0},-5^{0},+5^{0}$ and $+10^{0}$ so that the angle of incidence of the probe beam at maximum diffraction efficiency were $5.618^{0}, 8.894^{0}, 15.312^{0}$ and $18.421^{0}$ respectively inside the photopolymer layers. Angular selectivity curves were fitted to the data from which we obtained the positions of the Bragg peaks and the thicknesses of the gratings [12]. Hence the angular detuning can be corrected for any initial Bragg detuning of the probe He-Ne beam.

\section{Results and discussion}

Fig 2(a) shows the diffraction efficiency growth curve of $+5^{0}$ slanted gratings for different intensities but the same total exposure. From the graph it is clear that the polymerization rate increases with intensity. The fitted angular selectivity curves for slant angle $+5^{0}$ are shown in Fig 2 (b), as plots of normalized diffraction efficiency versus angle inside the photopolymer layer, where the position of the Bragg peak of the unslanted grating is $+15.312^{0}$ inside the photopolymer layer for 1000 lines $/ \mathrm{mm}$. The angles inside the photopolymer layer were calculated using Snell's law. From these graphs one can determine the shifts in the Bragg peaks and hence calculate the shrinkage occurring in the photopolymer layer. It is clear from the graphs that the shift of Bragg peak is greater for exposure at lower intensity and longer exposure time which is $1 \mathrm{~mW} / \mathrm{cm}^{2}$ and $80 \mathrm{sec}$.

Fig.3 shows the shift of the Bragg peak versus slant angle for three different intensities. Using Eq. (1) one can fit the linear dependence of the $\tan \left(\phi_{1}\right)$ versus $\tan \left(\phi_{0}\right)$ in order to obtain the shrinkage of the material. Fig. 4 shows the tangent of initial slant angle versus tangent of final slant angle. The 
slope gives the percentage shrinkage of the material. The $\%$ shrinkages evaluated from the fitted curves are $1.9 \%, 1.3 \%$ and $1 \%$.

The experimental findings of increased shrinkage at lower intensities of exposure can be correlated with the fact that at lower illumination intensity the polymer molecules formed are likely to be larger. The reason for this is that the rate at which the free radicals are generated is smaller and thus the volume concentration of free radicals is lower. This leads to a lower rate of termination and the ultimate result is the creation of longer polymer chains each comprising a larger number of monomer molecules. It is known that the polymerised material has higher density due to the morphology of the polymer material made of entangled polymer molecules. For a greater extent of polymerization the final density and thus the total dimensional change of the layer will be greater. The experimental results imply that a careful consideration should be given to the recording conditions when the effect of shrinkage must be avoided. Higher intensities are recommended but the maximum achievable refractive index modulation seems to be lower in these cases (Fig.2a).

\section{Conclusions}

Transmission diffraction gratings of spatial frequency 1000 lines/mm were recorded in an acrylamide based photopolymer film having $60 \pm 5 \mu \mathrm{m}$ thickness at constant exposure and different intensities and exposure times. We have obtained the Bragg curves and Kogelnik's coupled wave theory was used to fit the angular selectivity curves of the gratings. We have obtained grating thickness and final slant angles from the Bragg curve and hence calculated the shrinkage. The material shows linearity in Bragg detuning in slanted gratings and higher shrinkage is noted for recording with lower intensity and longer time of exposure. The next step is the modification of the photopolymer material in order to decrease the shrinkage due to photopolymerisation. We are studying the effect of incorporating different nanoparticles in the material. The results will be presented elsewhere.

\section{Acknowledgments}

This work was supported by a scholarship provided by the Technological Sector Research: Strand IPost-Graduate R\&D Skills Programme. The authors would like to acknowledge the School of Physics, DIT for technical support. 


\section{References}

[1] O V Sakhno, L M Goldenberg, J Stumpe and T N Smirnova, Surface modified ZrO2 and TiO2 nanoparticles embedded in organic photopolymers for highly effective and UV- stable volume holograms: Nanotechnology 18 (2007) 105704 (7pp).

[2] Lou Shou-Jun, Liu Guo-Dong, He Qing -Sheng, Wu Min- Xian, Jin Guo-Fan, Shi Meng Quan, Wang Tao, Wu Fei Peng, Holographic grating formation in dry photopolymer film with shrinkage: CHINESE PHYSICS. 13, 1428-1431 (2004).

[3] S. Martin, C. A. Feely, and V. Toal, Holographic recording characteristics of an acrylamide-based photopolymer: Appl.Opt. 36, 5757-5768 (1997).

[4] F. T. O’Neill, J. R. Lawrence, J. T. Sheridan, Thickness variation of self-processing acrylamidebased photopolymer and reflection holography, Opt. Eng. 40, 533-539 ( 2001)

[5] A. Belendez, I. Pascual, and A. Fimia, Efficiency of Thick Phase Holograms in the Presence of Shear-type Effects Due to Processing, J. Mod. Opt. 39, 889-899 (1992).

[6] L. Solymar and D. J. Cooke, Volume Holography and Volume Gratings: Academic Press, New York, 1981.

[7] Gallo J.T and Verber C. M, Model for the effects of material shrinkage on volume holograms: Applied Optics..33, 6796 (1994)

[8] C. Neipp and A. Beléndez, S. Gallego, M. Ortuño and I. Pascual, J. T. Sheridan, Angular responses of the first and second diffracted orders in transmission diffraction grating recorded on photopolymer material, OPTICS EXPRESS. 11, 1835$1843(2003)$.

[9] Hosam Sherif, Izabela Naydenova, Suzanne Martin, Colm McGinn and Vincent Toal, "Characterization of an acrylamidebased photopolymer for data storage utilizing holographic angular multiplexing,” J. Opt. A: Pure Appl. Opt. 7 , 255-260, (2005) .

[10] S. Gallego, M. Ortuno, C. Neipp, C. Garcia, A. Belendez, I. Pascual, Overmodulation effects in volume holograms recorded on photopolymers, Optics Communications. 215, 263-269, (2003).

[11] Mingju Huang, Huawen Yao, Zhongyu Chen, Lisong Hou, and Fuxi Gan, The changes of holographic characteristics of photopolymer induced by temperature, Chinese Optics Letters.1, 41-43 (2003).

[12] H. Kogelnik, Coupled wave theory for thick hologram gratings, Bell. Syst Techn. J., 48, p. 2909 (1969) 


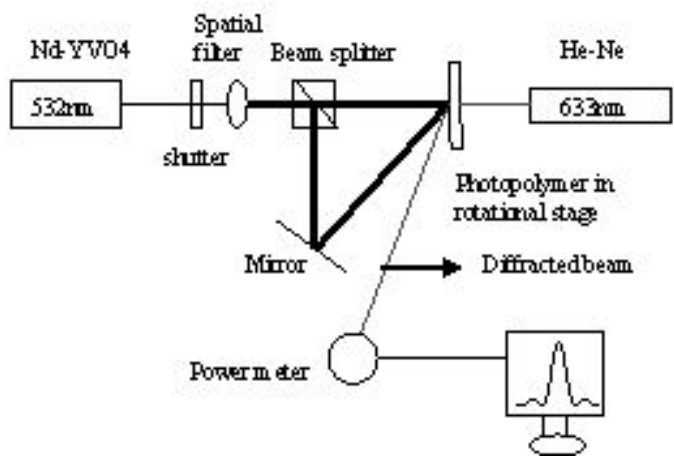

Fig. 1 Optical set-up for recording transmission phase holographic gratings

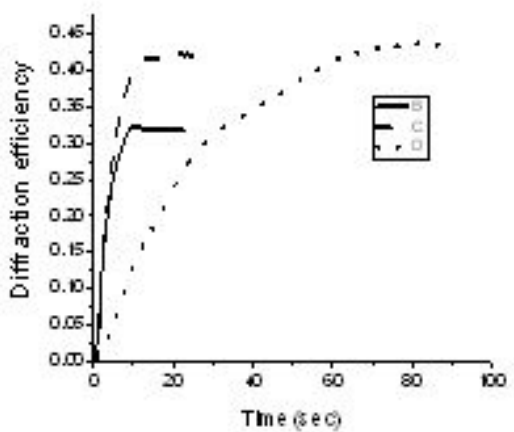

(a)

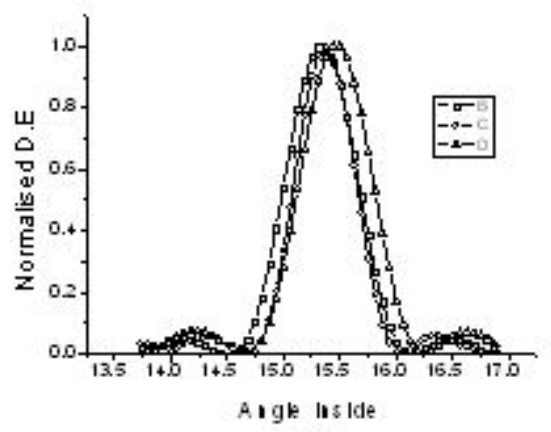

(b)

Fig. 2 Diffraction efficiency growth (a) and angular selectivity curves (b) for gratings recorded at B- $10 \mathrm{~mW} / \mathrm{cm}^{2}$, $8 \mathrm{sec}$; C- $5 \mathrm{~mW} / \mathrm{cm}^{2}, 16 \mathrm{sec}, \mathrm{D}-1 \mathrm{~mW} / \mathrm{cm}^{2}, 80 \mathrm{sec}$. The corresponding peak positions in (b) are B- $15.349^{0}$, C$15.359^{0}$, D- $15.401^{0}$. 


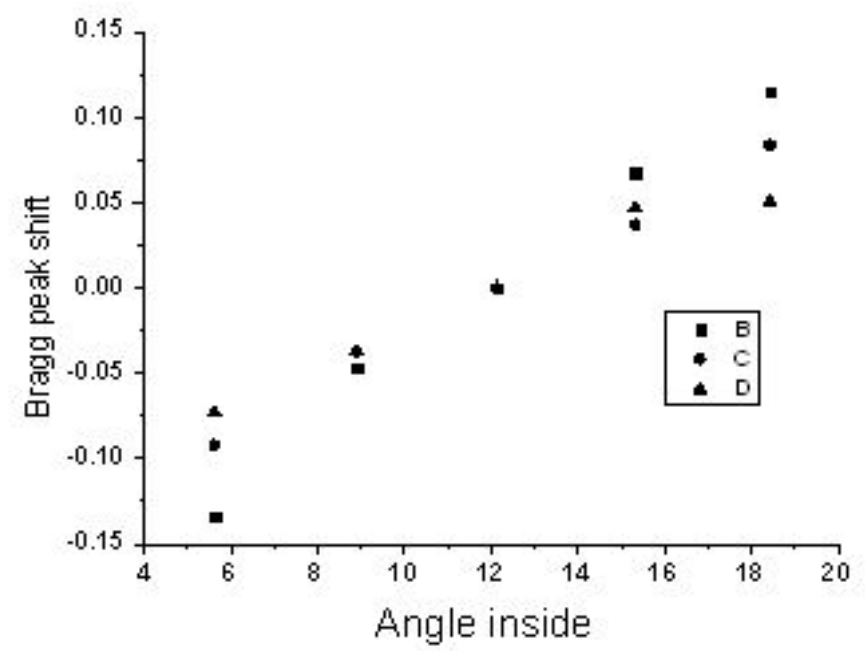

Fig. 3 Bragg peak shift for gratings recorded at a set of different slant angles versus the recording intensity. The total exposure was kept constant at $80 \mathrm{~mW} / \mathrm{cm} 2 ; \mathrm{B}-10 \mathrm{~mW} / \mathrm{cm}^{2}, \mathrm{C}-5 \mathrm{~mW} / \mathrm{cm}^{2}, \mathrm{D}-1 \mathrm{~mW} / \mathrm{cm}^{2}$

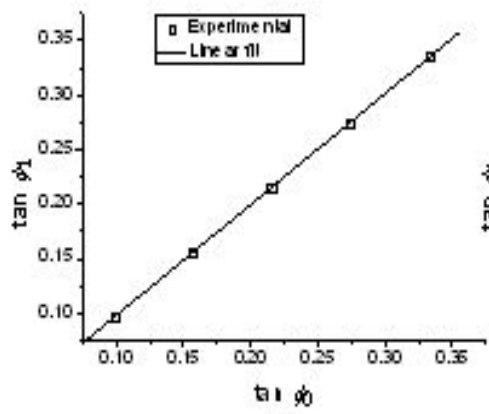

(a)

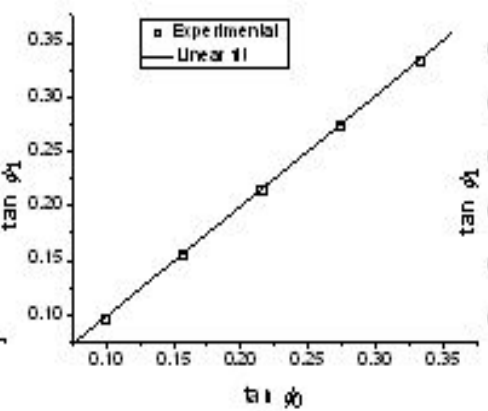

(b)

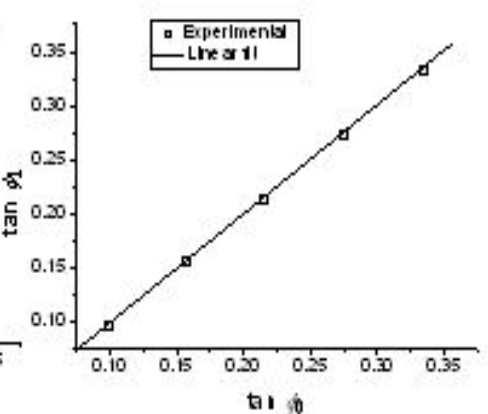

(c)

Fig. 4 Dependence of the new slant angle on the initial slant angle for three different intensities. The total exposure was kept constant at $80 \mathrm{~mW} / \mathrm{cm}^{2}$. (a) $1 \mathrm{~mW} / \mathrm{cm}^{2}$ (b) $5 \mathrm{~mW} / \mathrm{cm}^{2}$ (c) $10 \mathrm{~mW} / \mathrm{cm}^{2}$ 